\title{
On the Modelling of Rotational Effects in the Lower Convective Region of the Sun
}

\author{
H. Cavus* \\ Canakkale Onsekiz Mart University, Arts \& Science Faculty, Physics Department 17100, Canakkale, Turkey \\ A.I. Karafistan ${ }^{\dagger}$ \\ Near East University, Faculty of Engineering, Yakin Dogu Bulvari, Lefkosa, via Mersin 10, Turkey
}

(Received on 3 September, 2009)

\begin{abstract}
The purpose of this work is to study effects of differential rotation in the lower convective region of the Sun. Similar to MHD case; the governing equations are separated in variables, allowing numerical integration in this layer. This algorithm facilitates solutions of more complicated systems, with less computing time. Two different known rotation profiles are used in order to fit the model. The fitting procedure is accomplished by making use of sphericity and density shape parameters related to the rotation profiles. It is also shown that the most important feature of rotation is to break the spherical symmetric distribution of density in this layer. As in the MHD effects found before, differential rotation changes considerably the density of the reference model for both cases.
\end{abstract}

Keywords: Hydrodynamics-Sun: general-Sun: interior-Sun: rotation-Sun: fundamental parameters.

\section{INTRODUCTION}

It is well known that similar to other astronomical objects the Sun also rotates around its axis. However, considering the Sun as plasma rather than a rigid body, different parts of the Sun are thought to rotate at different rates. This gives rise to differential rotation which is mainly caused by the interaction of rotation and convective motions [1]. In a previous study magnetic field effects have been investigated in a lower convective region nominated as LCR [2, hereafter Paper I]. LCR is the region located in the vicinity of the base of convection zone. Here we provide a two-dimensional (2-D) model for this layer which can be used in inferring some physical parameters in the presence of differential rotation.

One of the great achievements of helioseismology was to map out the internal rotation of the Sun. It revealed that the convective zone was rotating differentially, with negligible angular velocity variation in depth, and a radiative interior in quasi-uniform rotation. These studies had shown the necessity to take rotation into account as an additional parameter in the solar models. Thus, inference of the solar internal rotation could be possible by measuring the frequency differences between the Doppler-shifted sound waves. A detailed review of recent progress in obtaining the solar rotation can be found in the work of [3]. Sun spots motions provide another tool about the rotation rate of the solar surface. It is well known that the Sun's rotation rate decreases with increasing latitude, so that it is slowest at the poles. However, the interior of the Sun does not spin the same way as the surface. Solar physicists believe that the Sun's inner core and the radiative zone rotate more like a solid body, contrary to the outer convective zone rotating differentially. The intermediate shear layer with different rotation properties was named by [4] as the solar tachocline.

On the other hand, physical effects in the overshoot region, which includes the LCR, are known to play an important role in solar evolution studies. A uniform rotation rate of $\Omega=$ $2.7 \times 10^{-6} s^{-1}$ of the radiative interior (including part of the

\footnotetext{
*Electronic address: h_cavus ecomu.edu.tr

${ }^{\dagger}$ Electronic address: akarafistan@neu.edu.tr
}

LCR) corresponds to the sidereal period of 27 days, which also characterizes the turnover time motions at the base of convective zone. According to [5] the angular velocity can be considered almost constant with respect to depth in the convective region. It decreases by $31 \%$ from the value of 2.9 $\times 10^{-6} s^{-1}$ at the equator towards the poles. Similarly, [6] state that besides the lower rotation at the poles, a latitudinal dependence of $\Omega$ exists.

From the complete study of a differentially rotating Sun, [7] concluded that the exact shape critically depends both on the rotation law from the subsurface to the tachocline. As before, we have named the study zone extending in the layer $0.703 R_{\odot}-0.723 R_{\odot}$ as LCR, which includes the $10^{4} \mathrm{~km}$ deep overshoot layer [8]. The centre of the solar tachocline is situated around $0.693 R_{\odot} \pm 0.002 R_{\odot}[9$, hereafter CTST $]$ and our study area is located above it. Behaviour of this region, especially near the base of the convective zone (used as $r_{C}$ ), has been studied vigorously by [10]. In their work radial and latitudinal variations of the solar rotation rates are obtained from the observed solar oscillations. They conclude that the helioseismological inverse models do not possess enough resolution for studying the dynamics as well the thickness of the tachocline. Therefore more elaborate direct models still need to be developed. [11], considering interaction between a global rotation profile and Reynolds stresses described the role of latitudinal shear instabilities in the solar tachocline. We can cite many authors concerned about influence of rotation (e.g. [6], [12], [13], [14]), as well as the magnetic dynamo effects [15].

The main aim of this study was to employ the thin shell model of the LCR, previously used in the MHD modelling in Paper I, with regard to the differential rotation effects instead of the magnetic one. By successive transformations, the governing fluid equations were separable in the spatial variables for the steady state. This allowed the numerical integration of physical state variables for parametric values of differential rotation rates (independent of time). The solar model of [16, hereafter JCD96], which was calibrated with respect to the solar oscillations, is used as a reference model. We have then constructed a 2-D hydrodynamic modelling including differential rotation in the LCR. Distributions of the most important physical parameters related to the rotational 
effects are investigated by the formalism explained in more detail in the following section. Numerical solutions of density, shear and vorticity are presented for two rotation profiles. Results including figures and tables are given in Section 4 and compared with some research works in Section 5. Different aspects of the works cited above will be compared in the last section with our results.

\section{PHYSICAL AND MATHEMATICAL METHOD}

\subsection{Model Assumptions}

In the present study, an algorithm suitable for a parametric representation of a differentially rotating thin LCR model is developed. Coriolis and centrifugal effects due to rotation are not considered as a perturbation to any other model representing this layer. Rather, we consider a rotation profile in the poloidal $(r, \theta)$ direction which introduces azimuthal $(\phi)$ symmetry in the spherical coordinates.

This model is imposed to satisfy the following physical assumptions;

- In the radiative part, resistivity and viscosity are sufficiently small allowing an ideal hydrodynamical representation.

- Similar to [4] a geostrophic flow is assumed in the upper part of the LCR, with negligible viscosity. This is true for sufficiently small Ekman and Rossby numbers, related to viscosity and Coriolis effects in the tachocline [11]. Taking into account these properties, only rotational terms are to be considered in the momentum transfer equation.

- On the other hand, slow meridional components driven by buoyancy could exist ([1], [17]). For the small viscosity values in our zone of interest, the small meridional flow components [18] are disregarded here, as it is also done in the work of [19].

- Similar to Paper I, the sound speed in the thin LCR being nearly constant $\left(2.284 \times 10^{5} \mathrm{~m} / \mathrm{s}\right)$, isothermality is assumed for the equation of state.

With these assumptions in mind, we intend to search parametric solutions for LCR, which will provide the necessary initial conditions for the future dynamical modelling.

\subsection{Basic Equations}

After these assumptions basic formulation of the hydrodynamical equations used by many authors, is applied to the solar LCR. As explained before the fluid motion is dominated by the Coriolis and centrifugal forces, since the corresponding Ekman number is asymptotically small. Then, we investigate the influence of differential rotation from the solution of the fundamental equations described by [20]:

$$
\begin{gathered}
\frac{\partial \rho}{\partial t}+\nabla \cdot(\rho v)=0 \\
\rho \frac{d v}{d t}=-\nabla p+\rho g-2 \rho(\Omega \times v)-\rho \Omega \times(\Omega \times r)
\end{gathered}
$$

The above equations expressing mass continuity and the momentum transfer are to be solved in the LCR. The physical parameters $\rho, v, p$ and $\Omega$ are used for the mean values of density, linear velocity, pressure and angular velocity respectively. With isothermality, the sound speed $c_{s}$ relating pressure $p$, to the density $\rho$ is given by:

$$
p=\rho \frac{c_{s}^{2}}{\gamma}
$$

The ratio of specific heats, $\gamma=1.664$. Due to nonlinearity, analytical solutions of (1)-(3) being not possible without further simplifying assumptions; we proceed with a steady-state equilibrium case, excluding time.

\subsection{Steady-State equilibrium}

Introducing an axially symmetric rotation profile to the mechanical equilibrium configuration, characteristics of the azimuthal flow is investigated in a thin LCR. Assumptions given above are taken into account for the fluid motion which is dominated by centrifugal and Coriolis forces. Starting from the steady-state equilibrium case, special solutions of the momentum transfer (2) are searched for an axially symmetric angular velocity in the $r$ and $\theta$ directions of the spherical coordinates [21]:

$$
\Omega=\omega \cos \theta \mathbf{e}_{r}-\omega \sin \theta \mathbf{e}_{\theta}
$$

where $\omega$ indicates the angular speed of the differential rotation. As in Paper I, for a toroidal flow, which is independent of the azimuthal $\phi$ direction (i.e. axially symmetric), the above equations are reduced to a steady-state:

$$
\rho(v . \nabla) v=-\nabla p+\rho g-2 \rho(\Omega \times v)-\rho \Omega \times(\Omega \times r) .
$$

With the use of (4), (5) can be separated in the $r$ and $\theta$ coordinates:

$$
\begin{gathered}
\rho \frac{\mathrm{v}^{2}}{r}=\frac{\partial p}{\partial r}+\rho g-2 \rho v \omega \sin \theta-\rho \omega^{2} r \sin ^{2} \theta \\
\rho \frac{\mathrm{v}^{2} \cot \theta}{r}=\frac{1}{r} \frac{\partial p}{\partial \theta}-2 \rho v \omega \cos \theta-\rho \omega^{2} r \sin \theta \cos \theta .
\end{gathered}
$$

We should note that $\mathrm{v}$ is used for the scalar $\phi$ components of the linear velocity.

\section{NUMERICAL SOLUTIONS}

We use the similar type of separation of variables outlined in [22] and Paper I, to rewrite (6) and (7) in a dimensionless form. After some mathematical manipulation, they are combined to a second order differential equation for density, to be solved by our algorithm developed for MAPLE 9.5 (Maplesoft, Waterloo, Ontario, Canada).

\subsection{Description of the model equations including differential rotation in the $L C R$}

We consider the hydrodynamical modelling of differential rotation in the LCR, covering a layer of $x=0.703$ to 0.723 of 
the solar radius. Normalising the radial coordinate $(r)$ as in [23]:

$$
r=R_{1}+z d .
$$

The parameters of (8) being selected so that $R_{1}$ is located at the bottom of the LCR (i.e. $\mathrm{z}=0$ ), $d$ represents the thickness of LCR, with $z$ expressing the dimensionless height. The values of $z=0$ and 1 correspond respectively to the bottom and top of this shell as shown in Figure 1.

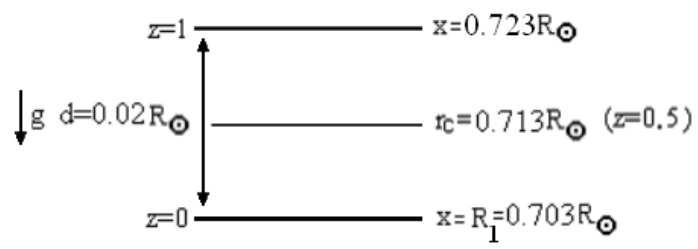

FIG. 1: Thin shell representation of the LCR in the Sun [2].

The position parameter $r_{C}$ indicates the location of the base of the convective zone. LCR is defined in the vicinity of $r_{C}$ as mentioned above. Therefore, its location is chosen at the midpoint of the LCR, in order to make the problem more tractable. The relative thickness $\eta$ in the thin shell approximation is again supposed to be small:

$$
\eta=\frac{d}{R_{1}}<<1
$$

We can similarly rescale (6) and (7) with the substitution of the dimensionless variables $\rho^{\prime}, \mathrm{v}^{\prime}, p^{\prime}, g^{\prime}$ and $\omega^{\prime}$. These are related to the physical parameters by the expressions given in equation 10 of Paper I, with the addition of the rotation parameter $\omega$, related to the dimensionless $\omega^{\prime}$ by:

$$
\omega=\frac{U}{R_{1}} \omega^{\prime}
$$

If the maximum magnitude of $\omega^{\prime}$ is set to 1 , then the normalisation velocity $\mathrm{U}$ can be determined. Finally, rewriting (6) and (7) in dimensionless form and using the same way of separation of variable for all physical parameters ( $\mathrm{v}^{\prime} \omega^{\prime}$, $\rho^{\prime}$ and $\left.v^{\prime}\right)$ as in [2], a general second order equation for $R$ is obtained:

$$
\frac{c_{s}^{\prime 2}}{\gamma} \frac{d^{2} R}{d z^{2}}+\frac{d R}{d z}\left[g^{\prime}-\frac{2 \mu c_{s}^{\prime 2}}{\gamma(1+\eta z)}\right]+\frac{2 \eta \mu c_{s}^{\prime 2}}{\gamma(1+\eta z)^{2}} R=0 .
$$

$c_{s}^{\prime}$ is the dimensionless sound speed, i.e. $c_{s}^{\prime 2}=\gamma p^{\prime} / \rho^{\prime}$, and $R$ expresses the radial dependence of density (for details of derivation see Paper I). This equation will be solved in the presence of differential rotation after defining the appropriate boundary conditions.

\subsection{Boundary conditions and model parameters including rotation}

A hydrodynamical model of a thin LCR becomes even more complicated with the inclusion of magnetic and rota- tional effects. We attempt to construct a reliable model in this region with high spatial resolution by imposing the following boundary condition at the lower boundary $\mathrm{z}=0$ :

$$
R(z)=1, \quad \frac{d R}{d z}=-\kappa .
$$

The constant $\kappa$, satisfying the correct value of JCD96 $(\kappa=$ $\eta \gamma g^{\prime} / c_{s}^{\prime 2}=0.222$ ), which describes the hydrostatic equilibrium condition and the LCR density value of $203 \mathrm{~kg} / \mathrm{m}^{3} \mathrm{ob}-$ tained as in Paper I. Then some parametric solutions of (11) for two different angular speed profiles, available from helioseismology will be obtained as follows.

\section{Case 1:}

[25, hereafter WBL] studied the inference of solar internal rotation from frequency splitting data from the profile of:

$$
\omega(r, \theta)=2 \pi\left\{A-B\left[1-\left(\frac{r}{R_{\odot}} \sin \theta\right)^{2}\right]-C\left[1-\left(\frac{r}{R_{\odot}} \sin \theta\right)^{2}\right]^{2}\right\}
$$

with $A=462, B=77$ and $C=42$.

Relationship between the rotation rate and the heat balance, in the upper part of the tachocline is estimated by [25] as $d \omega / d z \propto d T / d \theta$. Since we have assumed that a thin LCR should act as isothermal, there is no z-dependence of $\omega$ in this region. Further, [6] and [26] have found that the radial dependence of the angular speed has a constant value of $2.6 \times 10^{-6}$ $\mathrm{rad} / \mathrm{s}$ in the lower convective region, which is used in our region of interest. Then, using the rotation rate (13) in the (10) and Paper I we obtain the values of $K(z)=1, \mathrm{U}=1272 \mathrm{~m} / \mathrm{s}$ as Case 1 . The sphericity parameter $\mu$ and the density shape parameter $\delta$, described by Paper I. In Figure 2 the angular dependencies of $\omega$ for the WBL and our thin shell method are compared for the parametric values of $\mu=1.20, \delta=0.026$. It is seen that there is an acceptable fit between the values of $0.127 \pi$ and $0.870 \pi$ for $\theta$.

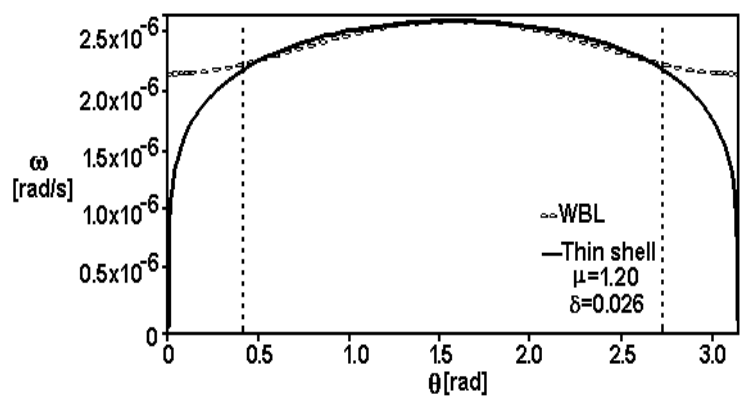

FIG. 2: Angular dependence of $\omega$ at the base of the convective zone for $\mu=1.20$ and $\delta=0.026$ (vertical dashed line shows the fitting limits)

\section{Case 2:}

From the helioseismic inversion of the observed LOWL data, CTST have derived a solar internal rotation rate for $r>0.5_{\odot}$ as below:

$$
\omega(r, \theta)=\omega_{C}+\frac{1}{2}\left[1+\operatorname{erf}\left(\frac{r-r_{C}}{\mathrm{w}}\right)\right] \times\left(\omega_{S}(\theta)+\omega_{C}\right) .
$$

The latitudinal differential rotation $\omega_{S}$ in this expression is defined as:

$$
\omega_{S}(\theta)=\omega_{E Q}+c_{1} \cos ^{2} \theta+c_{2} \cos ^{4} \theta
$$




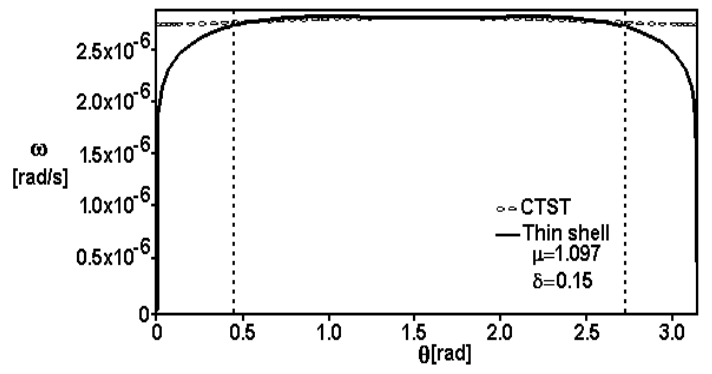

FIG. 3: Angular dependence of $\omega$ at the base of the convective zone for $\mu=1.097$ and $\delta=0.15$.

with parameters $\omega_{C}=2 \pi \times 432.8 n H z, \omega_{E Q}=2 \pi \times 460.7 n H z$, $c_{1}=-62.69 \mathrm{nHz}, c_{2}=-67.13 \mathrm{nHz} ; r_{C}=0.713 R_{\odot}$ and $w=0.025 R_{\odot}$. This time $\omega(r, \theta)$ obtained from this profile has almost a constant value of $2.8 \times 10^{-6} \mathrm{rad} / \mathrm{s}$ in the LCR, with respect to the radial distance. As in Case $1 K(z)=1$, but $\mathrm{U}=1370 \mathrm{~m} / \mathrm{s}$. From the comparison of the angular dependencies of $\omega$ given by CTST and our thin shell method in Figure 3, we observe a rather well fit between $0.127 \pi$ and $0.870 \pi$ for the parametric values of $\mu=1.097$ and $\delta=0.15$.

\section{RESULTS}

Imposing the boundary conditions defined in (12) for the above two cases, we solve first the general second order differential (11) for the radial dependence of density denoted as $R(z)$. A 4th order Runge-Kutta integration scheme is employed for a step size of 0.001 for this purpose. After $R(z)$, dimensionless density distributions in the LCR are obtained and shown in Figures 4-5. Vorticity (Vo), as well as the linear and angular velocity shears are drawn as functions of $\mathrm{z}$ and $\theta$ in Figures 6-9. All $z$ and angular variations are plotted at the angular position of $\theta=\pi / 2$ and $z=0.5$, corresponding to the equator and bottom of the convection zone respectively.

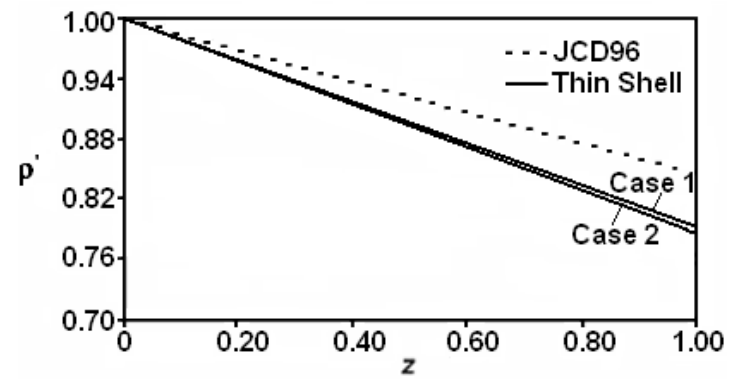

FIG. 4: Variations of the dimensionless density $\rho^{\prime}$, for JCD96 and the differentially rotating thin shell model.

In Figure 4 radial distribution of $\rho^{\prime}$ is represented for Cases 1,2 and JCD96. We notice that dimensionless density $\rho^{\prime}$ varies from 1 to 0.795 . This corresponds to a change of 20 $\%$ from the upper to the lower boundary of the LCR. Effect of rotation with respect to JCD96 is about $7 \%$ at the upper boundary. With the use of (3) and the density profile represented in this figure, the pressure scale height can also be found as $6.02 \times 10^{7} \mathrm{~m}$ and $6.04 \times 10^{7} \mathrm{~m}$ for Case 1 and 2 respectively, at the midpoint.

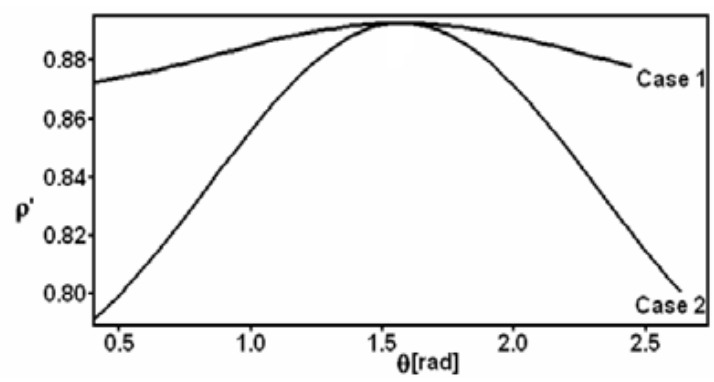

FIG. 5: Comparison of $\rho^{\prime}$ for Case 1 , Case 2 at $z=0.5$ as a function of $\theta$.

In Figure 5 the angular dependencies of the dimensionless density is given for the bottom of the convective zone. We notice that the dimensionless density has the same maximum value at the equator $(\theta=\pi / 2)$ but deviates about $8 \%$ at the Polar Regions. In addition, with increasing departures from sphericity (i.e. greater $\mu$ ), the LCR fluid density is seen to increase towards the poles.

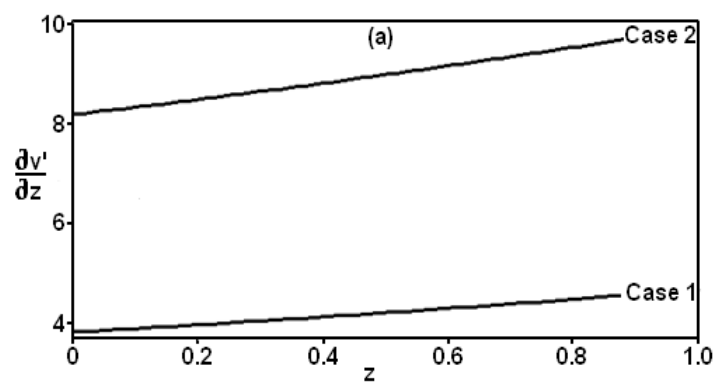

FIG. 6: Linear velocity shear in the LCR with respect to dimensionless height at $\theta=\pi / 2$.

From Figure 6, distribution of the linear velocity shear $\partial \mathrm{v}^{\prime} / \partial \mathrm{z}$ is seen to increase with $\mathrm{z}$, attaining greater values for Case 2. In Figure 7 the $d \ln \omega / d \theta$ variations of the angular velocity shear, which are related to the rotation rate itself are given as a function of the co-latitudinal angle $(\theta)$. For both cases it is noticed that this ratio is positive for the northern hemisphere $(\theta<\pi / 2)$ and negative for the southern one. The most important result for both cases is that there exists no shear in the angular velocity at the equator for this type of differential rotation. There is also a factor of two between the two cases at the extreme limits of $0.127 \pi$ and $0.870 \pi$ near the poles.

Additionally the components of vorticity exhibit an interesting feature. The radial component, shown in Figure 8, starts from a positive value in the northern hemisphere, vanishes at the equator and becomes negative in the southern part. This behaviour repeats more abruptly for Case 2. From 


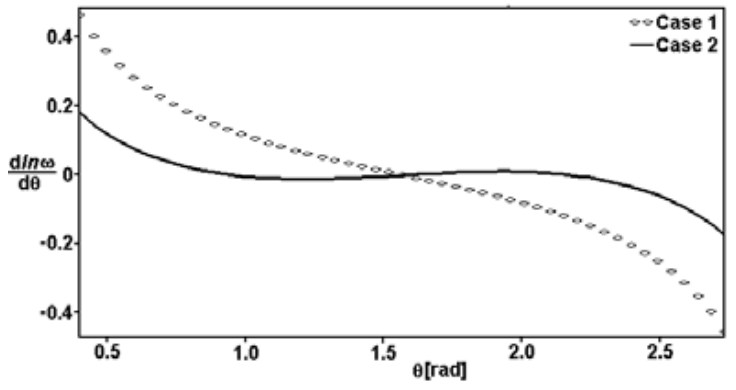

FIG. 7: Change of $\mathrm{d} \ln \omega / \mathrm{d} \theta$ (angular velocity shear) with respect to $\theta$ at at $z=0.5$.

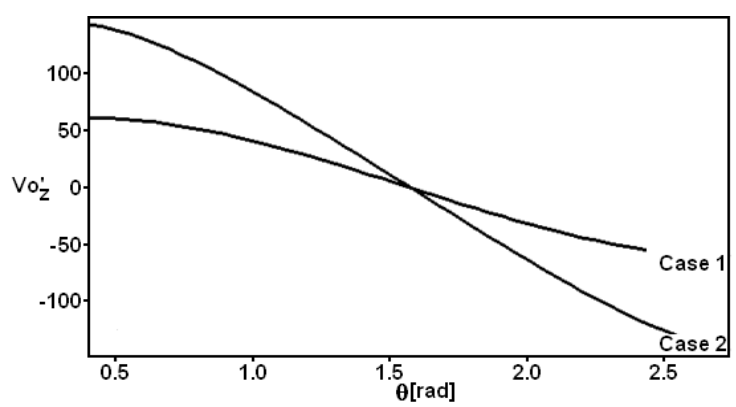

FIG. 8: Angular variations of radial component of $\boldsymbol{V o}$ at $z=0.5$.

the co-latitudinal component in Figure 9, we observe negative values for both cases with minima at the equator.

\section{DISCUSSIONS AND CONCLUSION}

Helioseismology has revealed that the convection zone in the Sun should be rotating differentially. However, there exists a thin tachocline layer, separating the radiative interior from the outer convective one. In this work we have concentrated on the hydrodynamical modelling of the LCR, including centrifugal and Coriolis terms due to some special rotation profiles. Indeed, viscosity and other secondary effects being neglected, this is not a differential rotation model ap-

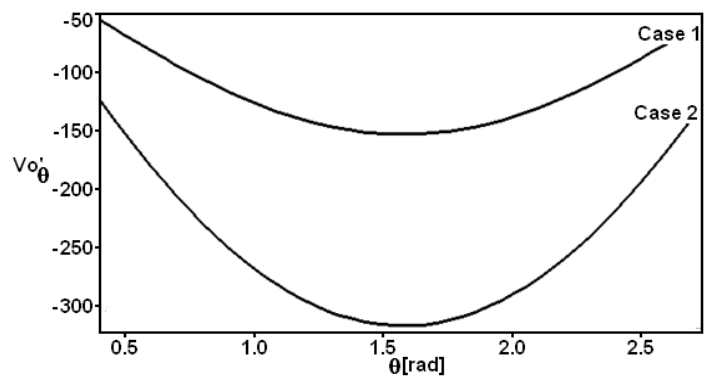

FIG. 9: Angular variations of co-latitudinal component of $\boldsymbol{V o}$ at $z=0.5$.

plicable to the whole convection zone in the Sun. As in Paper
I, which deals only with the MHD effects, we have developed an axially symmetric thin shell model, elaborating the presence of differential rotation for a Coriolis number around 2, corresponding to a rapid rotation [27].

We modelled effects of Coriolis and centrifugal forces in a layer of $0.703 R_{\odot}-0.723 R_{\odot}$, and integrated numerically by a step size of 0.001 , for some physical variables. Finally, density and other parameters related to rotation are obtained in dimensionless form from the solution of a general differential (11). Simulations relating effects of rotational forces within full spherical shells have revealed interesting behaviours for two known differential rotation profiles. The sphericity $(\mu)$ and density shape parameters $(\delta)$, describing behaviour of the specific rotation profile in the thin shell method, are calibrated with two other rotation profiles existing in solar literature (WBL and CTST). The angular dependencies of our rotation profiles (Figures 2 and 3 ) show resemblance with that of [6] in an outer layer.

With the special rotation profiles, we have found that there is a $20 \%$ difference in density at the lower and upper limits of the LCR (see Figure 4). This corresponds to an overall 7 $\%$ difference with respect to the non-rotating thin shell in the reference model (JCD96). This also corresponds to the same order of magnitude for the magnetised thin shell case (Paper I). Comparing the pressure scale height values of our model with that of [28], we find concordances about 99.6 and 99.3 $\%$ for Cases 1 and 2 respectively.

The rotation rate, which is found to be slower at the poles than the equator is comparable in behaviour to that of [6]. As stated further by [29], the generally cyclonic nature of vorticity is in good accordance with our parametric solutions for its components. While the radial one decreases from positive to negative values in both hemispheres, the angular one is negative, with a minimum at the equator for both cases. Similarly [11] also had shown that the radial component of vorticity reaches a maximum near the pole, diminishing to zero at the equatorial region (i.e. our $\theta=\pi / 2$ ). Another important result related to vorticity is its conservation [11] at the outermost boundaries for the angular positions (i.e. $0.127 \pi$ and $0.870 \pi$ ) in the Cases I and II. On the other hand, according to [12] if the angular velocity shear goes to zero (our case Figure 7), then the angular velocity should be constant in a certain region (Figure 2 and 3). These conditions are seen to be fulfilled from the figures indicated in parenthesis, with additionally vorticity transforming smoothly into a rigid body rotation (see Figure 8).

The changes in the linear velocity shear rate $\left(\partial \mathrm{v}^{\prime} / \partial \mathrm{z}\right)$ with respect to dimensionless height have a similar increasing tendency with that of [30] at the equatorial region. In their turbulence modelling, they found a linear change in the shear rate with respect to radius, corresponding to a maximum of $1.5 \times 10^{-5} s^{-1}$. Comparing this result to our Case 1 and 2 results, which are $3.80 \times 10^{-4}$ and $8.25 \times 10^{-4}$ respectively, we can conclude that the centrifugal and Coriolis forces are producing 10 times greater shear than the linear effects of turbulence with rotation.

We should again emphasize that this is not a perturbation method, but rather a parametric one, which can provide the necessary initial conditions for studies including time as an additional parameter. 
[1] E.R. Priest, Solar Magneto-Hydrodynamics, D.Reidel Publishing Company, Dordrecht, Holland (2000).

[2] H Cavus and A.I. Karafistan, MNRAS, 380, 142 (2007). (Paper I)

[3] P. Garaud P. and T. Rogers, in J. S. Richard, H. Guenter, G.M.Rebecca and A.T. Christopher eds., AIP Conference Proc. Vol. 948, Unsolved Problems in Stellar Astrophysics, p.237 (2007).

[4] E.A. Spiegel and J.P. Zahn, A\&A, 265, 106 (1992).

[5] D. Gough, in Hughes D.W., Rossner R., Weiss N.O., eds, The Solar Tacholine, Cambridge University Press (2007).

[6] A.S. Brun and Toomre J., ApJ, 570, 865 (2002).

[7] S. Godier and J.P. Rozelot, A\&A, 355, 365 (2000).

[8] J. H. M. M. Schmitt, R. Rosner and H.U. Bohn, ApJ, 282, 316 (1984).

[9] P. Charbonneu, S.Tomczyk, J. Schou and M.J. Thompson, 1998, ApJ, 496, 1015 (1998). (CTST)

[10] H.M. Antia, S. Basu and S.M. Chitre, MNRAS, 298, 543 (1998).

[11] P. Garaud, MNRAS, 324, 68 (2001).

[12] V.M. Canuto, ApJ, 497, L51 (1998).

[13] M.S. Miesch, ApJ, 586, 663 (2003)
[14] M.S. Miesch, A.S. Brun and J. Toomre, ApJ, 641, 618 (2006).

[15] M. Ossendrijver, A\&AR, 11, 287 (2003).

[16] J. Christensen-Dalsgaard et al., Sci, 272, 1286 (1996). (JCD96)

[17] E. Forgacs-Dajka and K. Petrovay, A\&A, 389, 629 (2002).

[18] L.L. Kitchatinov, Astronomy Reports, 48, 153 (2004).

[19] M. Rempel, M. Schssler and G. Toth, 2000, A\&A, 363, 789 (2000)

[20] A.R. Choudhuri, The Physics of Fluids and Plasmas, Cambridge University Press, Cambridge (1998).

[21] M.L. DeRosa, PhD Thesis, University of Colorado (2001)

[22] J. J. G. Lima, E.R. Priest and K. Tsinganos, A\&A, 371, 240 (2001)

[23] M.S. Miesh and P.A. Gilman, Sol. Phys., 220, 287 (2004).

[24] P.R. Wilson, D. Burtonclay and Y. Li, ApJ, 470, 621 (1996). $(W B L)$

[25] D.O. Gough and M.E. McIntyre, Nat, 394, 755 (1998)

[26] M. Rempel, ApJ, 622, 1320 (2005).

[27] M.S. Miesch, ApJ, 562, 1058 (2001).

[28] M. Dikpati, P.A. Gilman and M. Rempel, ApJ, 569, 680 (2003).

[29] A.S. Brun, M.S. Miesch and J. Toomre, ApJ, 614, 1073 (2004).

[30] E. Schatzman, J.P. Zahn and P. Morel, A\&A, 364, 876 (2000). 